\title{
sensors
}

ISSN 1424-8220

(C) 2002 by MDPI

http://www.mdpi.net/sensors

\section{Study on pH Effect in Process of an Entero-gastric Fiber-optic Sensor Design}

\author{
Chen Guo-ping ${ }^{1 * *}$, Xia Rong-min ${ }^{1}$, Gong $\mathrm{Jun}^{2}$ and Shou Wen-de ${ }^{1}$ \\ ${ }^{1}$ Department of Biomedical Engineering, Shanghai Jiao Tong University, Shanghai 200030, China. \\ Tel.86-21-62932830. Fax 86-21-52540266. E-mail: gpchenapple@ hotmail.com \\ ${ }^{2}$ Department of Digestion, Second Hospital of Xi' an Jiaotong University, Xi' an 710004,China \\ * Author to whom correspondence should be addressed.
}

Received: 28 October 2002 / Accepted: 15 November 2002 / Published: 30 November 2002

\begin{abstract}
The $\mathrm{pH}$ effect on design of a fiber optic sensor newly developed for monitoring entero-gastric reflux is investigated. The research has been carried out by utilizing the sensor to measure characteristic absorption of bilirubin standard solution (used as a calibrator) at different $\mathrm{pH}$ values from about 1 to 8 . The results show explicitly that the estimation error according to the calibration curves with $\mathrm{pH}<7$ is significantly lower than that at alkaline $\mathrm{pH}$ values. Compared with the in vitro experimental data, the optimum $\mathrm{pH}$ range for entero-gastric reflux analysis is proposed to be between 5-6. The possible reasons for estimation error of the fiber optic sensor are given in detail.
\end{abstract}

Keywords: Fiber optic sensor, Bilirubin, pH, Entero-gastric reflux

\section{Introduction}

Entero-gastric reflux is the return of the duodenal contents back into stomach and sometimes even up to esophagus. More and more experimental and clinical evidences suggest that entero-gastric reflux may play an important role in dyspepsia, gastric ulcer, gastritis, gastric cancer, esophageal mucosal injury, or other pathological changes in digestive tract [1].

It is necessary to find a useful way for the clinic to monitor entero-gastric reflux in situ and continuously for a 24-hour or longer period. Traditional analytical methods need time-consuming sample preparation, careful separation and spectrophotometric procedure and are not suitable for in vivo clinical analysis. Because of its remote and fast sensing, fiber-optic sensors developed recently makes it possible to meet the demand. 
This kind of fiber optic sensor needs to be made portable and lightweight. And the sensing system should avoid the cumbersome and expensive spectrophotometers or optical power meters. Moreover the electric supply should ensure the sensor to operate for long-operational detection in situ. P.Bechi first reported the fiber optic sensor for ambulatory entero-gastric reflux analysis and it has been applied in clinical analysis [2].

Using the fiber optic sensor, Francesco Baldini et al. examined the relationship between $\mathrm{pH}$ of bile samples and its absorption by the in vitro analysis [3]. However, $\mathrm{pH}$ values may influence the enterogastric fiberoptic sensor in two aspects: $\mathrm{pH}$ effect on biological samples in clinical analysis and on standard bilirubin solution as a calibrator in design of the sensor. There has been no description concerned with the latter.

In this paper we design an improved fiber-optic sensor for analysis of entero-gastric reflux and study the $\mathrm{pH}$ effect in design of the sensor. In the study, bilirubin standard solutions with $\mathrm{pH}$ ranging from 1.24 to 7.76 are used to set up linear regression equations expressing the relationship between bilirubin absorbance and its concentration. The results are compared with that obtained by the in vitro analysis of bilirubin present in bile. The appropriate $\mathrm{pH}$ range for the sensor is proposed. The discussions about $\mathrm{pH}$ effect on bilirubin estimation of the kind of sensors [3-6] are given and possible reasons of their discrepancies are presented.

\section{Principle and Experimental Set-up of the Sensor}

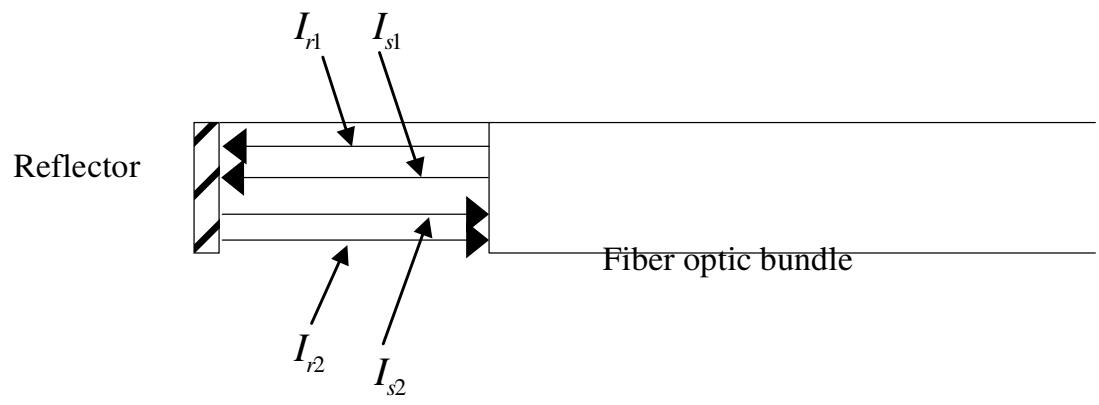

Figure 1. The principle of the double-beam compensation. The sensing tip is dipped into sample solution containing an absorbing substance.

The fiber optic sensor performs on the principle of double-beam compensation [7] as illustrated in Figure 1. The reference and signal lights ( $I_{r 1}$ and $I_{s 1}$ respectively) transmit out of the fiber-optic bundle and go through the bilirubin solution. The intensities of reflected lights $\left(I_{r_{2}}\right.$ and $\left.I_{s 2}\right)$ transmitting back into the bundle are found to be as follows:

$$
\begin{aligned}
& \frac{I_{r 2}}{I_{s 2}}=\frac{I_{r 2}}{I_{s 1}} \cdot \frac{I_{s 1}}{I_{s 2}} \approx \frac{I_{r 1}}{I_{s 1}} \cdot \frac{I_{s 1}}{I_{s 2}} \\
& A=\log \frac{I_{r 2}}{I_{s 2}}=\log \frac{I_{r 1}}{I_{s 1}}+\log \frac{I_{s 1}}{I_{s 2}}=\alpha+\varepsilon L c=\alpha+k c
\end{aligned}
$$


where $\alpha=\log \frac{I_{r 1}}{I_{s 1}}, I_{r 2} \approx I_{r 1}, \log \frac{I_{s 1}}{I_{s 2}}=\varepsilon L c=k c$ (Lambert-Beer law), $L$ is the optic length, $c$ is the concentration of an absorbing substance and $\varepsilon$ is the molar absorptive coefficient. As for a certain fixed sensor, $\alpha, k$ and $\varepsilon$ can be regarded as constants.

Figure 2 outlines the optical sensing system used for analytical research. Two super bright LEDs (470 nm as signal light and $595 \mathrm{~nm}$ as reference light) are utilized as optical sources. A special bandpass filter is positioned before the signal LED. The lights emitting from the LEDs are coupled to one of the arms of the Y-model fiber-optic bundle with core/cladding diameter 300/350 $\mu \mathrm{m}$. The beams transmit through the bundle to the distal end and then are reflected from a mirror. The reflected beams, traveling into and then out of the other arm of the bifurcated bundle, are converted into electric signals by a photodiode receiver. After A/D conversion, the signals are sampled and stored by a microprocessor and will be analyzed later.

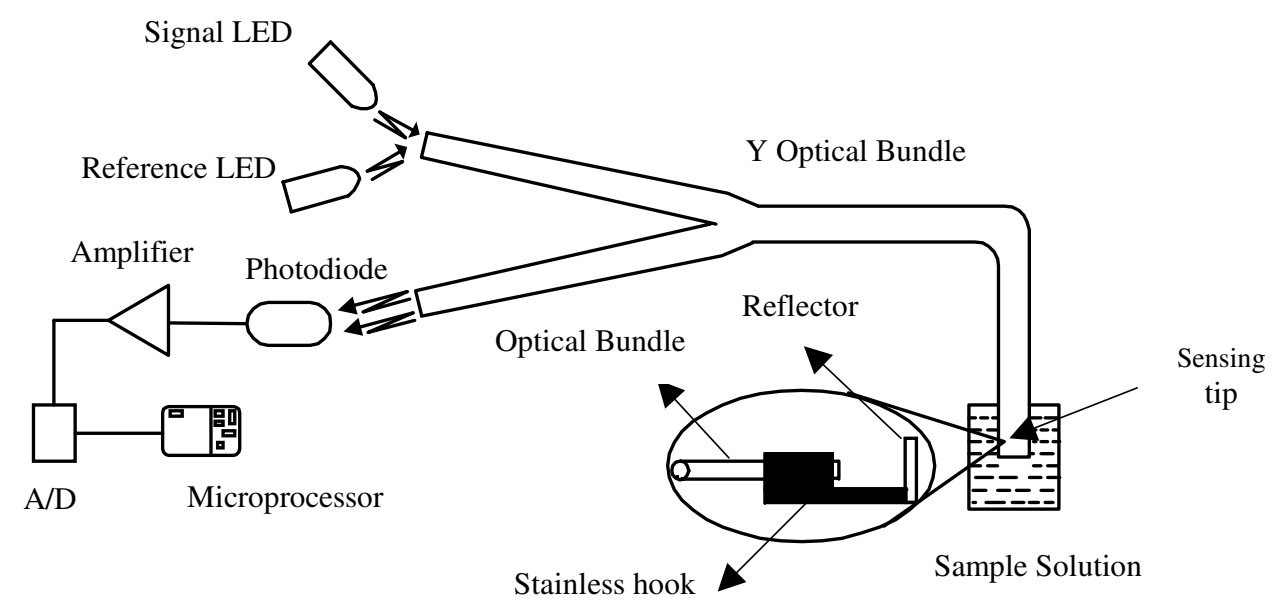

Figure 2. Experimental set-up of the sensing system.

\section{Materials and Methods}

Bilirubin stock solution $\left(10 \mathrm{mg} / \mathrm{dl}, 1.71 \times 10^{-4} \mathrm{~mol} / \mathrm{l}\right)$, which is=fabricated by dissolving bilirubin in serum and then lyophilized, is purchased from Shanghai Institute of Biological Products. Its $\mathrm{pH}$ is measured by REX pHS-3C pH meter. All the standard solutions with controlled $\mathrm{pH}$ values are prepared by careful dilution with $1 \mathrm{~mol} / \mathrm{l} \mathrm{HCl}$ solution. In this way, the bilirubin solution can be as close as possible to physiological environment, suggested by Francesco Baldini [3].

Bile samples are drawn directly from gallbladder of the patient. They are kept in dark and at less 0 temperature when they are not optically analyzed. The lower concentrations of the biological samples are adjusted by using $\mathrm{HCl}$ solution at $\mathrm{pH}$ 1.26. The quantitative determination of total bilirubin of bile samples is carried out by the Vanden Bergh method (Clinical Diagnostic Reagent, Total Bilirubin Reagent Kit, 20011201, Shanghai Institute of Biological Products of Ministry of Public Health).

All the samples are stored in glass beakers wrapped by aluminium foil. The analytical procedure is performed in the dark to avoid light exposure. Each sample is measured by the sensor and $\mathrm{pH}$ meter respectively. 


\section{Results and Discussion}

Two of the most concerns in sensors are their sensitivity and stability. The calibration curves of bilirubin standard solution at $\mathrm{pH} 1.26$ and 7.46 respectively are constructed in Figure 3 . The absorbance/concentration curves exhibit a reasonable linear dynamic range up to $10 \mathrm{mg} / \mathrm{dl}$ $\left(1.71 \times 10^{-4} \mathrm{~mol} / \mathrm{l}\right)$ with a correlation coefficient of 0.9998 . The linear range seems to be better than that reported by P.Becchi [2] and comparable to that by the spectrophotometric analysis [6]. The stability is checked by dipping the sensing tip into distilled water and measuring its absorbance every hour for 8 hours. It is found that the mean relative error is less than $2 \%$. The stability can fulfill the clinical analysis.

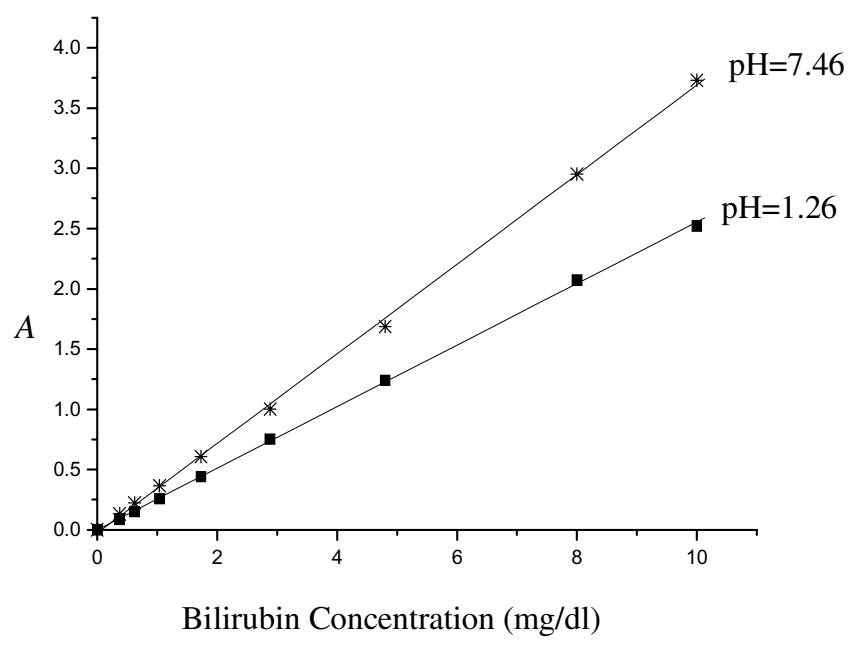

Figure 3. Absorbance $A$ vs. concentration of the bilirubin standard solution.

As is well known, gastric fluid is normally acidic with $\mathrm{pH}$ range 1-2. When $\mathrm{pH}$ increases over 4, the pepsin then loses its activity and digestive system may be damaged. But as for the patients with duodenogastroesophageal diseases, $\mathrm{pH}$ of gastric juice mixed with duodenal reflux or medications may fluctuate from acid about at $\mathrm{pH} 1.0$ to alkaline at about 7.0. So it is necessary to study $\mathrm{pH}$ effect on concentration estimation when the fiber-optic sensors well designed are applied in clinical analysis.

For bilirubin (mono- and di-) glucuronide is not available in the market, unconjugated bilirubin $(\mathrm{Bu})$ or bilirubin conjugate-ditaurobilirubin (DTB) is widely used as calibrators in clinical analysis=of bilirubin. In the same way they are applied in design of the fiber optic sensors as calibrators. The relationship between $\mathrm{pH}$ and bilirubin absorption of the calibrator is associated with the accuracy of the fiberoptic sensor for clinical analysis.

Figure 4 and Figure 5 show the $\mathrm{pH}$ dependence on bilirubin calibrator absorbance by the fiberoptic sensor. Interestingly, a very weak $\mathrm{pH}$ dependence is apparent in the acid environment. But at $\mathrm{pH}>7$ the calibration curve strays obviously from those obtained in acid solutions. The maximal relative error of concentration evaluation is about $15 \%$ as for $3 \mathrm{mg} / \mathrm{dl}$ in acid solution. As $\mathrm{pH}$ rises above 7 , the estimation error can be up to $36 \%$. Similar results are found in Figure 5, in which the 
absorbance is versus different concentrations of bilirubin at 10,8,4 and $0.8 \mathrm{mg} / \mathrm{dl}$ in the $\mathrm{pH}$ range 17.5. As expected, the curves fluctuate little at $\mathrm{pH}<7$ while drastic increase appears in the presence of alkaline $\mathrm{pH}$.

The relation between $\mathrm{pH}$ and the absorbance might be attributed to the N-B transformation of albumin conformation [8]. This can be found in the absorption spectra (Figure 6) by a fiber optic spectrophotometer (Ocean Optics PS 2000). In Figure 6 a red shift of about $25 \mathrm{~nm}$ for the absorption peak is observed over the $1.68-7.70 \mathrm{pH}$ range, and the shoulders become narrower or relative narrower as $\mathrm{pH}$ increases.

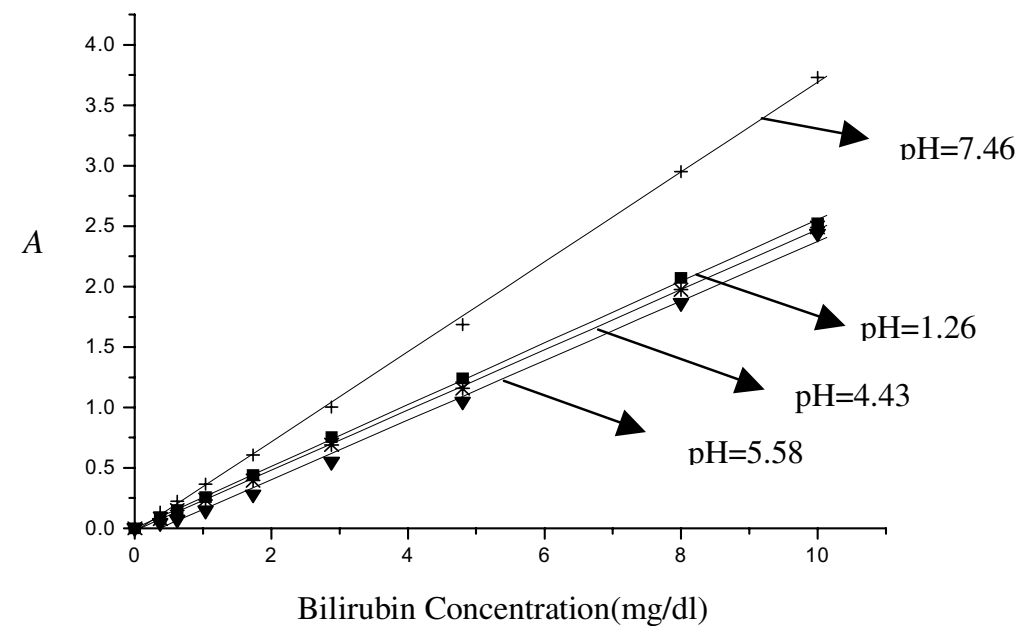

Figure 4. Bilirubin absorbance /concentration curves at different $\mathrm{pH}$.

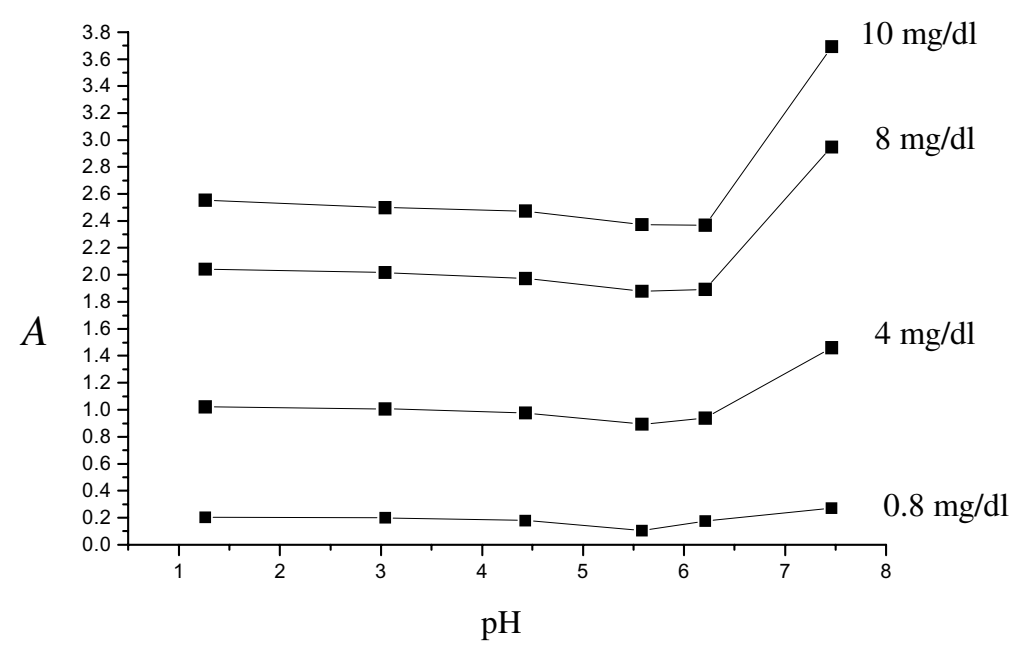

Figure 5. Absorbance of bilirubin with concentrations at $0.8,4,8$ and $10 \mathrm{mg} / \mathrm{dl}$ in the range of $\mathrm{pH}$ 1-8. The absorbance A is deduced from the regression lines obtained.

In vitro experimental results for the fiber optic sensor are shown in Figure 7. The linear regression lines at pH 1.26 and 6.21 are herein plotted together. Signal linearity with up to concentration of 10 
$\mathrm{mg} / \mathrm{dl}$ is achieved for the bile sample detection. The linear response is consistent with the dynamic linear range obtained by the standard bilirubin solution. Above the limit of $10 \mathrm{mg} / \mathrm{dl}$ the curve saturates obviously. Considering $\mathrm{pH}$ effect on standard calibration, it is easy to find that the line at $\mathrm{pH} 6.21$ is preferably used as the calibration curve in design of the fiber optic sensor. However the calibration (curve b) compared with sampling response (curve c) is still not very ideal. The estimation error as to absorbance 1.0 comes up to $33 \%$.

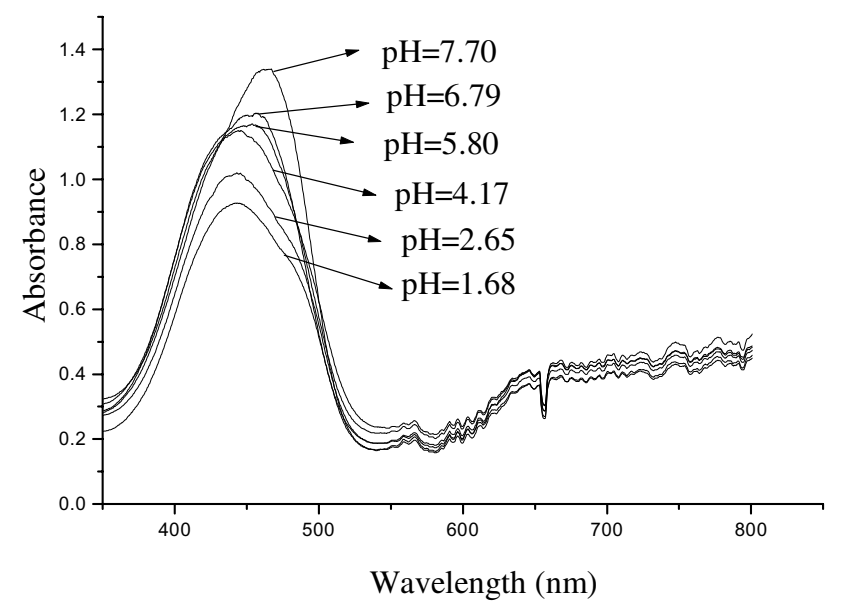

Figure 6. Absorbance spectra of bilirubin with different $\mathrm{pH}$ values.

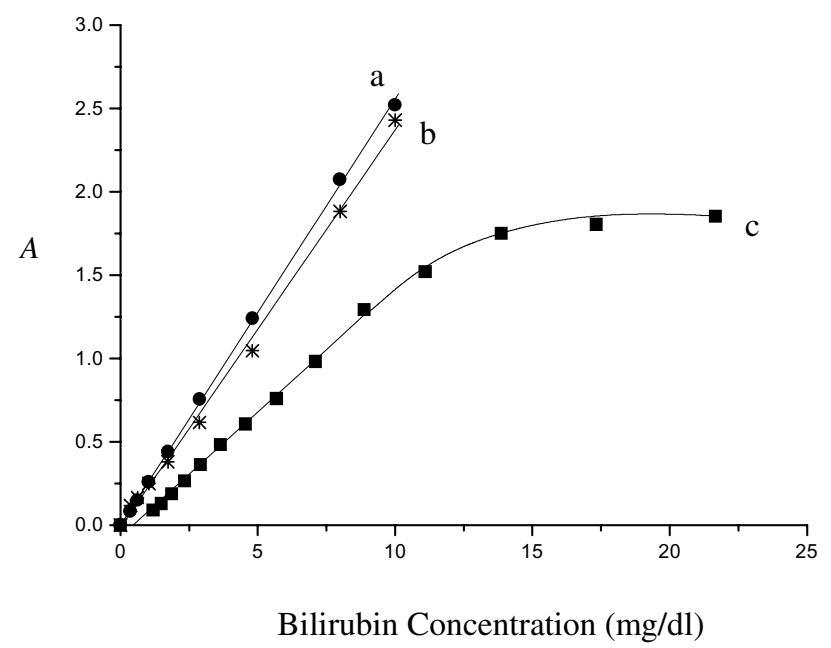

Figure 7. Comparison of the fiberoptic sensor response in bilirubin standard solution at $\mathrm{pH}$ 1.26 (curve a) and 6.21 (curve b) to that in bile mixture with $\mathrm{HCl}$ solution at $\mathrm{pH} 1.16$ (curve c).

There are a few reports concerning the relationship between $\mathrm{pH}$ and bilirubin absorbance detected by the commercial fiber optic sensor, Bilitec 2000. And they seem to have no consistency. It was reported by Werner K.H., et al [4] that there is about 30\% underestimation of bilirubin absorbance in acid compared to alkaline environment. And G.Champion et al. [5] suggested that an underestimation 
of up to $30 \%$ for $\mathrm{pH}<3.5$ occurred when using the Bilitec 2000. However, based on results of the analysis of bile by Bilitec 2000, Francesco Baldini, et al. [3] concluded that the maximum error in evaluation of bilirubin concentration was only about $13 \%$ instead of $30 \%$. Gong Jun, et al. [6] also provided some important information about $\mathrm{pH}$ and bile sample sensing by Bilitec 2000 .

Our results suggest that in acid solution $\mathrm{pH}$ has less effect on bilirubin absorbance than in alkaline environment. And even in acidic solution the relative error of concentration estimation corresponding to bilirubin absorbance 1.0 is more than $33 \%$.

The discrepancies discussed above may be explained by the complexity of compounds in biological samples. Bile sample is composed of conjugated bilirubin (diglucuronide and mono glucuronide), unconjugated bilirubin, cholalic acid, phospholipid, and other organic or inorganic components. Their complicated interaction with each other and the respective spectra of colorful constitutes make optic behavior of the bile sample much different from the bilirubin standard solution used as a calibrator for establishment a linear regression equation.

The choice of wavelength can also affect sensing accuracy. The maximal absorption wavelength of bilirubin solution used as a calibrator lies around $450 \mathrm{~nm}$. But the peak of absorption spectrum for the bile sample often shifts to $400 \mathrm{~nm}$ when $\mathrm{pH}$ is $<3$. So the estimation error is inevitable using the established calibration equation.

Another factor may be the precipitates resulting from bile compounds at lower $\mathrm{pH}$ value. The dissociation constants of conjugated bilirubin in bile are more than 3 . Some components of the bile sample become insoluble when mixed with gastric juice whose $\mathrm{pH}$ is always 1-2. Then such a condition does not sufficiently meet with the prerequisite for the validity of Lambert-Beer law.

\section{Conclusion and Outlook}

A new fiber optic sensor for analysis of entero-gastric reflux is designed. It is important to investigate $\mathrm{pH}$ effect on absorption of standard bilirubin solution effect in design of the sensor because it is concerned with its accuracy in clinical diagnosis. The results suggest that in acid environment $\mathrm{pH}$ effect on bilirubin absorbance is much smaller than in alkaline solution. As $\mathrm{pH}$ rises to alkaline $\mathrm{pH}$, the estimation error becomes unacceptable. Compared with biological analysis in vitro, the regression line established by bilirubin standard solution over the 5- $6 \mathrm{pH}$ range is even preferable.

It's still much difficult to measure bilirubin concentration precisely. The complexity of bile sample and inaccessibility of technology suitable to clinically analyze in situ and continuously may contribute to the difficulty.

In consideration of relationship between $\mathrm{pH}$ and bilirubin absorbance, a better method is to design a multiple fiber optic sensor that is integrated with bilirubin sensor and $\mathrm{pH}$ meter. In design of the sensor, it is possible to store standard calibration curves at different $\mathrm{pH}$ in a microprocessor. The sensor can measure bilirubin absorbance and $\mathrm{pH}$ values simultaneously and can be easy to detect accurately $\mathrm{pH}$ and bilirubin concentration at the same time. Anymore $\mathrm{pH}$ value of gastric juice is still an important item for clinical analysis. It will improve detectable rate of duodenogastric reflux (DGR) in clinical diagnosis. 


\section{Acknowledgements}

The authors would like to thank Liu Xu, Sun Jun-Xia, et al. in our laboratory for their valuable discussion. Professor $\mathrm{Xu}$ Yu-hong did us much favor during our analysis and is gratefully acknowledged here. This project was funded in part by National Natural Science Foundation of China under Grant No. 39970209.

\section{References}

1. Qizhang, W. Gastroesophageal Reflux Diseases; Tianjing Scientific and Technical Publisher: Hunan, 1994; pp. 52-60.

2. Bechi, P.; Falciai, R.; Baldiini, F. et al. A new fiber optic sensor for ambulatory entero-gastric reflux detection. Proceedings of Fiber Optic Medical and Fluorescent Sensors and Applications. 1992, 1648, 130-135.

3. Baldini, F.; Bechi, P.; Cianchi, P. et al. Analysis of the optical properties of bile. Journal of Biomedical Optics 2000, 5(3), 321-329.

4. Werner, K.H.; Kauer, M.D.; Patricio Burdiles, M.D.; Ireland, A.P. et al. Does Duodenal Juice Reflux Into the Esophagus of Patients With Complicated GERD? Evaluation of a Fiberoptic Sensor for Bilirubin. The AMERICAN JOURNAL OF SURGERY 1995, 169, 98-104.

5. Champion, G.; Richter, J.E.; Vaezi, M.F. et al. Duodenogastroesophageal Reflux: Relationship to $\mathrm{pH}$ and Importance in Barrett's Esophagus. Gastroenterology 1994, 107, 747-754.

6. Gong, J.; Zhang, R.; Luo, J.Y. et al. The effect of bile reflux on the intragastric pH. Journal of Xi'AN MEDICAL UNIVERSITY 2001, 22(1), 25-27.

7. Fan, Sh.F.; Chen, L.; Xiao, S. Sh. et al. Design and Development of Optical Fiber Sensor for Multipurpose Biochemical Sensing. Chinese Journal of Scientific Instrument 1999, 1, 52-59.

8. Heirwegh, K. P.M.; Brown, S.B. Bilirubin (translated into Chinese by Shen Tao \& Zhang Naijian). Union Publisher of Beijing Medical University and China Xiehe Medical University, 1991, pp. 102-107.

Sample Availability: Available from the authors.

(C) 2002 by MDPI (http://www.mdpi.net). Reproduction is permitted for noncommercial purposes. 